\title{
QUEEN'S UNIVERSITY
}

\section{POLYMER RESEARCH GROUP}

19 Division Street, Kingston, ON, K7L 3N6 Canada

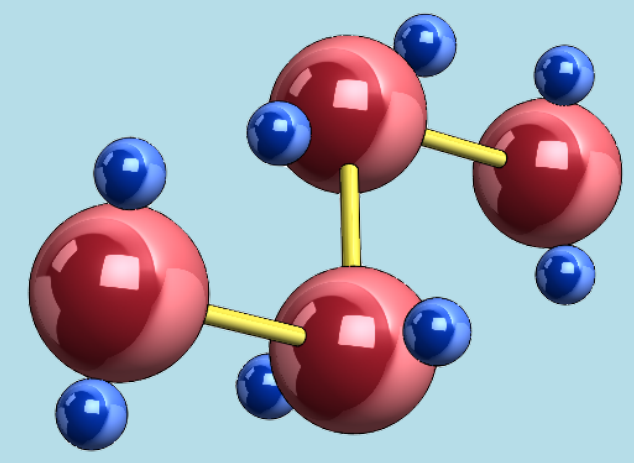

DEGRADATION IN CONE-PLATE RHEOMETRY

\author{
A.J. Giacomin ${ }^{1,2, *}$ and P.H. Gilbert ${ }^{1}$ \\ ${ }^{1}$ Polymers Research Group \\ Chemical Engineering Department \\ ${ }^{2}$ Mechanical and Materials Engineering Department \\ Queen's University \\ Kingston, ON K7L 3N6
}

This report is circulated to persons believed to have an active interest in the subject matter; it is intended to furnish rapid communication and to stimulate comment, including corrections of possible errors.

*Corresponding author (giacomin@queensu.ca)

QU-CHEE-PRG-TR--2018-45 


\title{
DEGRADATION IN CONE-PLATE RHEOMETRY
}

\author{
A.J. Giacomin $1,2,3 *$ and P.H. Gilbert ${ }^{1,3}$ \\ ${ }^{1}$ Chemical Engineering Department \\ ${ }^{2}$ Mechanical and Materials Engineering Department \\ ${ }^{3}$ Polymers Research Group \\ Queen's University \\ Kingston, Ontario, CANADA K7L 3N6
}

\begin{abstract}
We analyze quantitatively the oxidative degradation of a sample in a cone-plate rheometer, as oxygen diffuses inward, radially, from the free boundary. We examine rheometer error mitigation by means of nitrogen blanketing, and also, of cone-plate partitioning. We arrive at exact analytical expressions for the oxygen concentration, and thus, for the degradation rate. We then integrate this rate over time to get the amount of oxygen reacted as a function of radial position and time in the degrading sample. To illustrate the usefulness of our analytical expressions, we provide two worked examples investigating the effect of nitrogen blanketing and cone-plate partitioning. We find that, though nitrogen blanketing always produces less degradation, its benefits are limited for short times. Additionally, cone-plate partitioning provides a simpler solution and allows samples to be run for longer times without degradation compromising measurement, even in samples initially saturated with oxygen. We close by considering the effect of antioxidants.
\end{abstract}

Keywords: cone-plate rheometry, polymer, degradation, antioxidant

* Corresponding author (giacomin@queensu.ca). 


\section{CONTENTS}

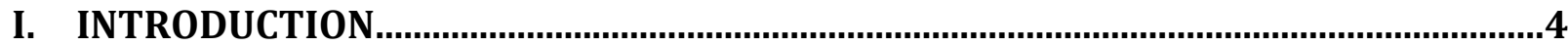

II. STEADY DEGRADATION IN AIR BATH .....................................................................

a. Total Oxidation ..............................................................................................................

III. UNSTEADY DEGRADATION WITH NITROGEN BLANKETING..................................... 7

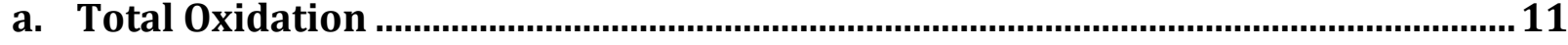

IV. UNSTEADY DEGRADATION WITHOUT NITROGEN BLANKETING ........................... 11

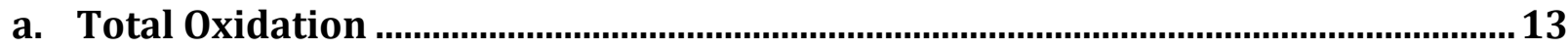

V. WORKED EXAMPLE: NITROGEN BLANKETING .................................................... 13

VI. WORKED EXAMPLE: CONE-PLATE PARTITIONING …............................................... 14

VII.UNSTEADY OXIDATION WITH ANTIOXIDANT DOPING ......................................... 14

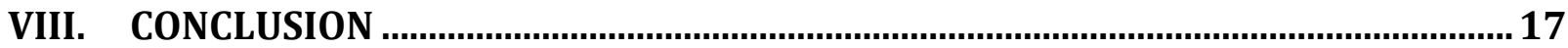

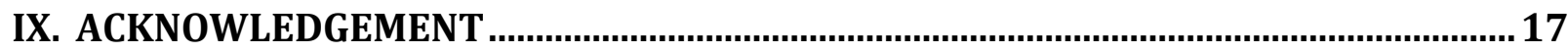

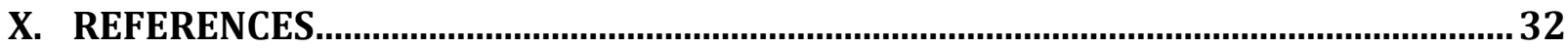

\section{FIGURES}

Figure 1: Sketch of sample in cone-plate rheometer without cone-plate partitioning, defining spherical coordinates and boundaries. .................................................20

Figure 2: Sketch of sample in cone-plate rheometer with cone-plate partitioning........21

Figure 3: Steady state dimensionless concentration profiles from Eq. (29) for $\kappa=0.1,1,10$.

Figure 4: Dimensionless concentration profiles versus radius, from Eq. (86), for five different times with a dimensionless rate constant of $\kappa=0.1 \ldots \ldots \ldots \ldots \ldots \ldots \ldots \ldots \ldots \ldots . . .23$

Figure 5: Dimensionless concentration profiles versus radius, from Eq. (86), for five

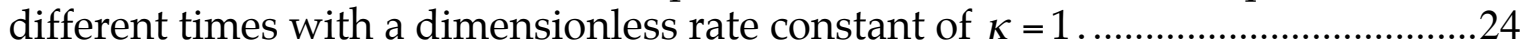

Figure 6: Dimensionless concentration profiles versus radius, from Eq. (86), for five

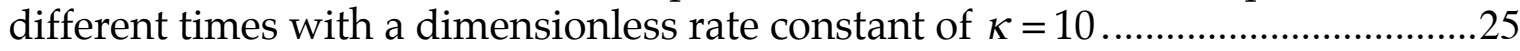

Figure 7: Dimensionless concentration profiles versus radius, from Eq. (104), for five different times with a dimensionless rate constant of $\kappa=3.93$ and an initial to rim

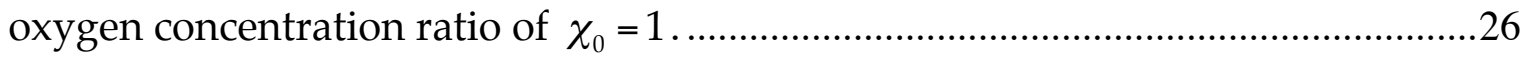

Figure 8: Dimensionless concentration profiles versus radius, from Eq. (105), for five different times with a dimensionless rate constant of $\kappa=3.93$ and an initial to rim oxygen concentration ratio of $\chi_{0}=1.2$.

Figure 9: Dimensionless concentration profiles versus radius, from Eq. (104), for five different times with a dimensionless rate constant of $\kappa=3.93$ and an initial to rim oxygen concentration ratio of $\chi_{0}=0.80$. 
Figure 10: Dimensionless concentration profiles versus time, from Eq. (89) and (108), for three different radii with a dimensionless rate constant of $\kappa=3.93$ and an initial to rim oxygen concentration ratio of $\chi_{0}=1$, where the solid and dashed curves correspond to the sample exposed to oxygen and the sample blanketed with nitrogen.

Figure 11: Dimensionless degradate concentration profiles versus radius, from Eq. (89) and (108), for five different times with a dimensionless rate constant of $\kappa=3.93$ and an initial to rim oxygen concentration ratio of $\chi_{0}=1$, where the solid and dashed curves correspond to the sample exposed to oxygen and the sample blanketed with nitrogen.

Figure 12: Pseudo-steady state dimensionless antioxidant (dashed) and doublyinflecting oxygen (solid) concentration profiles and the steady state oxygen concentration profile (dotted) versus radius, from Eq. (117)-(120) and Eq. (29), with $\alpha_{I}=0.32$ and dimensionless rate constants of $\kappa=3.93$ and $\kappa_{\mathrm{NA}}=13.0$, where $\chi_{\mathrm{N} 0}=10$.

\section{TABLES}

Table I: Dimensional Variables 18

Table II: Dimensionless Variables and Groups 


\section{INTRODUCTION}

This paper focuses on the particular source of error in cone-plate rheometry brought about by oxidative degradation of the polymer sample. This problem arises in the characterization of molten plastics, for instance, when the air bath exposes the sample free boundary to atmospheric oxygen. About the latter, Dealy and Wissbrun (Subsection 7.9.4 of [1]) observe "in cone-plate and parallel disk rheometers, the free surface at which degradation and composition changes first occur is at the outside radius where it has the maximum effect on the measured torque and normal force." We focus particularly on the cone-plate geometry, a nearly homogeneous flow field that is popular for rheological characterization [2,3]. Figure $\mathbf{1}$ illustrates this rheometer geometry for steady shear.

One particular incarnation of the cone-plate fixture, called cone-plate partitioning, measures the torque over just the middle of the sample $[4,5,6,7,8]$. By middle, we mean the portion of the sample circumscribed by a partitioning gap. Only the material within this gap contributes to the torque. We provide an sketch of a cone-partitionedplate rheometer geometry undergoing steady shear in Figure 2, where the inner partition has radius, $R_{P}$. One unintended advantage of such cone-plate partitioning is that it suppresses error due to oxidative degradation, by distancing the part of the sample contributing to the torque from the free boundary. In this paper, we analyze how well this distancing of the oxygen source from the measurement boundary can suppress error due to oxidative degradation.

When inserting a sample into a rheometer, one can either first remove its dissolved oxygen under vacuum or not. In Section II, we consider a sample that is initially devoid of oxygen, and in Section III, initially saturated. In Section II, we consider the steady state oxygen concentration that develops when the free boundary is exposed to air, and in Section III, to nitrogen.

Sometimes, polymer degradation is unavoidable during rheological characterization. For small-amplitude oscillatory shear flow measurements, a method has been devised for correcting for polymer degradation (see Subsection 19.2.3.2 of [1]).

Throughout this work we treat samples subject to oxidative degradation following a reaction that is first order in the concentration of oxygen, $c_{A}$. Both the initiation and propagation steps of oxidative polymer degradation, for instance, are typically first order in $c_{A}$ [9]:

$$
\begin{aligned}
& \mathrm{RH}+\mathrm{O}_{2} \rightarrow \mathrm{R} \bullet+\mathrm{HO}_{2} \cdot \\
& \mathrm{R} \bullet+\mathrm{O}_{2} \rightarrow \mathrm{RO}_{2}
\end{aligned}
$$

In this work we focus on the amount of oxygen consumed by the degradation process, and not on the rich diversity of reactions that can happen next (see Oxidative

Degradation, Chemistries in [9]).

The total amount of polymer degradative oxidation is given by:

$\mathrm{d}=-\int_{0}^{t} R_{A} d t$

where $\mathrm{d}[=] \mathrm{mol} \mathrm{L}^{-3}$ and where:

$R_{A}=-k^{\prime \prime \prime} c_{A}$

so that: 


$$
\begin{aligned}
\mathrm{d} & =-\int_{0}^{t}-k^{\prime \prime \prime} c_{A} d t \\
& =k^{\prime \prime \prime} \int_{0}^{t} c_{A} d t
\end{aligned}
$$

We complete our analysis with two worked examples in Sections V and VI to determine when it is best to use a nitrogen blanket and to investigate the benefits of cone-plate partitioning.

\section{STEADY DEGRADATION IN AIR BATH}

In this section, we solve for the steady state concentration profile that develops in the nearly isothermal degrading sample in an air bath. The sample is subject to oxidative degradation following a reaction that is irreversible and first order in the concentration of oxygen, $c_{A}$.

We first consider the steady state, supposing that:

$c_{A}=c_{A}(r)$

with the atmospheric boundary:

$c_{A}(R, \theta)=c_{A R}$

apex symmetry:

$\frac{\partial c_{A}(0, \theta)}{\partial r}=0$

and impermeable walls:

$\frac{\partial c_{A}\left(r, \frac{\pi}{2}\right)}{\partial \theta}=\frac{\partial c_{A}\left(r, \theta_{c}\right)}{\partial \theta}=0$

We simplify our equation of continuity (Eq. (B.4-3) of [10]), as follows:

$$
\begin{aligned}
& c\left(\frac{\partial x_{A}}{\partial t}+v_{r} \frac{\partial x_{A}}{\partial r}+\frac{v_{\theta}}{r} \frac{\partial x_{A}}{\partial \theta}+\frac{v_{\phi}}{r \sin \theta} \frac{\partial x_{A}}{\partial \phi}\right) \\
& =-\left[\frac{1}{r^{2}} \frac{\partial}{\partial r}\left(r^{2} J_{A r}^{*}\right)+\frac{1}{r \sin \theta} \frac{\partial}{\partial \theta}\left(J_{A \theta}^{*} \sin \theta\right)+\frac{1}{r \sin \theta} \frac{\partial J_{A \phi}^{*}}{\partial \phi}\right]+x_{B} R_{A}-x_{A} R_{B} \\
& 0=\frac{-1}{r^{2}} \frac{\partial}{\partial r}\left(r^{2} J_{A r}^{*}\right)+R_{A}
\end{aligned}
$$

using the standard substitutions for the mole-based equation found at the end of Section B.10 in [10], where $R_{A}[=] \mathrm{mol} / L^{3} / t$. We assume that the concentration of oxygen remains small relative to the polymer allowing for simplification of the reaction term in Eq. (10). For first order oxidative degradation:

$0=\frac{-1}{r^{2}} \frac{\partial}{\partial r}\left(r^{2} J_{A r}^{*}\right)-k^{\prime \prime \prime} c_{A}$

where $c_{A}[=] \mathrm{mol} / L^{3}$ and where $k^{\prime \prime \prime}[=] t^{-1}$.

We next eliminate the radial flux in terms of the molar concentration using Fick's law:

$J_{A r}^{*}=-\mathcal{C} \mathcal{O}_{A B} \frac{\partial x_{A}}{\partial r}=-\mathscr{D}_{A B} \frac{\partial \mathcal{C}_{A}}{\partial r}$ 
where:

$C x_{A} \equiv C_{A}$

to get:

$0=\mathscr{D}_{A B}\left[\frac{1}{r^{2}} \frac{\partial}{\partial r}\left(r^{2} \frac{\partial c_{A}}{\partial r}\right)\right]-k^{\prime \prime \prime} c_{A}$

Since all derivatives are with respect to $r$ :

$\frac{1}{r^{2}} \frac{d}{d r}\left(r^{2} \frac{d c_{A}}{d r}\right)-\frac{k^{\prime \prime \prime}}{\mathscr{O}_{A B}} c_{A}=0$

or:

$\frac{1}{\alpha^{2}} \frac{d}{d \alpha}\left(\alpha^{2} \frac{d\left(c_{A} / c_{A R}\right)}{d \alpha}\right)-\kappa^{2}\left(\frac{c_{A}}{c_{A R}}\right) \equiv 0$

where:

$\kappa^{2} \equiv \frac{k^{\prime \prime \prime} R^{2}}{\mathscr{O} \mathscr{N}_{A B}}$

is the second Damköhler number, $\mathrm{Da}_{\mathrm{II}}=\kappa^{2}$, and where:

$\alpha \equiv \frac{r}{R}$

Eq. (17) has the form:

$\frac{1}{x^{2}} \frac{d}{d x}\left(x^{2} \frac{d y}{d x}\right)-a^{2} y=0$

which has general solution given by Eq. (C.1-6a) of [10]:

$y=\frac{C_{1}}{x} \cosh a x+\frac{C_{2}}{x} \sinh a x$

so that:

$\chi(\alpha) \equiv \frac{c_{A}}{c_{A R}}=\frac{C_{1}}{\alpha} \cosh \kappa \alpha+\frac{C_{2}}{\alpha} \sinh \kappa \alpha$

which is subject to Eqs. (7) and (8). Differentiating Eq. (22), we find:

$\frac{d \chi}{d \alpha}=\left[\frac{\kappa \cosh \kappa \alpha}{\alpha}-\frac{\sinh \kappa \alpha}{\alpha^{2}}\right] C_{2}+\left[\frac{\kappa \sinh \kappa \alpha}{\alpha}-\frac{\cosh \kappa \alpha}{\alpha^{2}}\right] C_{1}$

Evaluating Eq. (23) at the apex, in the limit as $r \rightarrow 0$, gives:

$0=[0] C_{2}+[-\infty] C_{1}$

and thus:

$C_{1}=0$

so that:

$\chi=\frac{C_{2}}{\alpha} \sinh \kappa \alpha$

which when subjected to Eq. (7):

${ }^{1} \alpha x \tau i v \alpha$ is Greek for radius. 
$1=C_{2} \sinh \kappa$

gives:

$C_{2}=\frac{1}{\sinh \kappa}$

so that:

$\chi=\frac{1}{\alpha} \frac{\sinh \kappa \alpha}{\sinh \kappa}$

which is the dimensionless steady state concentration profile of oxygen in the sample. Figure 3 illustrates the steady state concentration profiles for low, medium and high reaction rates, $\kappa$. As we expect, lower reaction rates $(\kappa=0.1)$ allow oxygen diffusion from the rim to dominate causing the sample to be fully saturated, whereas large reaction rates $(\kappa=10)$ deplete the sample interior of oxygen.

\section{a. Total Oxidation}

Evaluating Eq. (5) at steady state, gives:

$$
\begin{aligned}
\mathrm{d} & =c_{A} k^{\prime \prime \prime} \int_{0}^{t} d t \\
& =c_{A} k^{\prime \prime \prime} t
\end{aligned}
$$

and substituting Eq. (29) into Eq. (30) gives the total amount of degradative polymer oxidation at any position $r$ in the sample:

$$
\begin{aligned}
& \mathrm{d}=\frac{c_{A R}}{\alpha} \frac{\sinh \kappa \alpha}{\sinh \kappa} k^{\prime \prime \prime} t \\
& \frac{\mathrm{d}}{c_{A R}}=\frac{1}{\alpha} \frac{\sinh \kappa \alpha}{\sinh \kappa} \kappa \tau
\end{aligned}
$$

Eq. (31) relies on a steady state solution, Eq. (29), and thus neglects the initial condition and all transient behavior.

\section{UNSTEADY DEGRADATION WITH NITROGEN BLANKETING}

In this section, we treat the nearly isothermal sample initially saturated with air (with an oxygen concentration of $c_{A 0}$ ) and blanketed in nitrogen upon loading into the viscometer. The sample is subject to oxidative degradation following a reaction that is irreversible and first order in the concentration of oxygen, $c_{A}$. We solve for the unsteady oxygen concentration profile in the nearly isothermal degrading sample.

Here we improve upon Eq. (6) of Section II by supposing that:

$c_{A}(r, t)$

We further use the atmospheric boundary condition:

$c_{A}(R, t)=0$

for the nitrogen blanketing, with the initial condition:

$c_{A}(r, 0)=c_{A 0}$

for the initial oxygen saturation, and with apex symmetry: 
$\frac{\partial c_{A}(0, \theta, t)}{\partial r}=0$

Our equation of continuity (Eq. (B.4-3) of [10]) simplifies as follows:

$c\left(\frac{\partial x_{A}}{\partial t}+v_{r} \frac{\partial x_{A}}{\partial r}+\frac{v_{\theta}}{r} \frac{\partial x_{A}}{\partial \theta}+\frac{v_{\phi}}{r \sin \theta} \frac{\partial x_{A}}{\partial \phi}\right)$

$=-\left[\frac{1}{r^{2}} \frac{\partial}{\partial r}\left(r^{2} J_{A r}^{*}\right)+\frac{1}{r \sin \theta} \frac{\partial}{\partial \theta}\left(J_{A \theta}^{*} \sin \theta\right)+\frac{1}{r \sin \theta} \frac{\partial J_{A \phi}^{*}}{\partial \phi}\right]+x_{B} R_{A}-x_{A} R_{B}$

so that:

$c \frac{\partial x_{A}}{\partial t}=\frac{-1}{r^{2}} \frac{\partial}{\partial r}\left(r^{2} J_{A r}^{*}\right)+R_{A}$

where $R_{A}[=] \mathrm{mol} / L^{3} / t$ and where:

$c x_{A} \equiv c_{A}$

For first order oxidative degradation:

$\frac{\partial c_{A}}{\partial t}=\frac{-1}{r^{2}} \frac{\partial}{\partial r}\left(r^{2} J_{A r}^{*}\right)-k^{\prime \prime \prime} c_{A}$

where $c_{A}[=] \mathrm{mol} / L^{3}$ and where $k^{\prime \prime \prime}[=] t^{-1}$. Eliminating the fluxes using (Eq. (B.3-7) of [10]):

$J_{A r}^{*}=-c \mathscr{O}_{A B} \frac{\partial x_{A}}{\partial r}=-\mathscr{O}_{A B} \frac{\partial \mathcal{C}_{A}}{\partial r}$

with Eq. (39), we get:

$\frac{\partial c_{A}}{\partial t}=\mathscr{O}_{A B}\left[\frac{1}{r^{2}} \frac{\partial}{\partial r}\left(r^{2} \frac{\partial c_{A}}{\partial r}\right)\right]-k^{\prime \prime \prime} c_{A}$

We proceed by non-dimensionalizing as follows:

$\mathrm{X} \equiv \frac{c_{A}}{c_{A 0}}$

$c_{A 0} \frac{\partial \mathrm{X}}{\partial t}=c_{A 0} \mathscr{O}_{A B}\left[\frac{1}{r^{2}} \frac{\partial}{\partial r}\left(r^{2} \frac{\partial \mathrm{X}}{\partial r}\right)\right]-c_{A 0} k^{\prime \prime \prime} \mathrm{X}$

$\frac{\partial \mathrm{X}}{\partial t}=\mathscr{Q}_{A B}\left[\frac{1}{r^{2}} \frac{\partial}{\partial r}\left(r^{2} \frac{\partial \mathrm{X}}{\partial r}\right)\right]-k^{\prime \prime \prime} \mathrm{X}$

and using Eq. (19):

$\frac{\partial \mathrm{X}}{\partial t}=\frac{\mathscr{D}_{A B}}{R^{2}}\left[\frac{1}{\alpha^{2}} \frac{\partial}{R \partial \alpha}\left(R^{2} \alpha^{2} \frac{\partial \mathrm{X}}{R \partial \alpha}\right)\right]-k^{\prime \prime \prime} \mathrm{X}$

$\frac{\partial \mathrm{X}}{\partial t}=\frac{\mathscr{O}_{A B}}{R^{2}}\left[\frac{1}{\alpha^{2}} \frac{\partial}{\partial \alpha}\left(\alpha^{2} \frac{\partial \mathrm{X}}{\partial \alpha}\right)\right]-k^{\prime \prime \prime} \mathrm{X}$

Introducing:

$\tau \equiv \frac{t \hat{O}_{A B}}{R^{2}}$ 
$\kappa^{2} \equiv \frac{k^{\prime \prime \prime} R^{2}}{\mathscr{O}_{A B}}[=] 1$

and using Eq. (19) gives:

$\frac{\partial \mathrm{X}}{\partial \tau}=\frac{1}{\alpha^{2}} \frac{\partial}{\partial \alpha}\left(\alpha^{2} \frac{\partial \mathrm{X}}{\partial \alpha}\right)-\kappa^{2} \mathrm{X}$

where $\chi(\alpha, \tau)$. Non-dimensionalizing Eqs. (34) through (36):

$\mathrm{X}(1, \tau)=0$

$\mathrm{X}(\alpha, 0)=1$

$\frac{\partial \mathrm{X}(0, \tau)}{\partial \alpha}=0$

Eq. (50) is thus subject to the initial condition, Eq. (51), and to boundary conditions Eqs. (52) and (53).

Supposing that:

$\mathrm{X}(\alpha, \tau) \equiv f(\tau) g(\alpha)$

so that from Eq. (51):

$f(\tau) g(1)=0$

and thus, we have the rim condition:

$g(1)=0$

and from Eq. (36):

$\frac{\partial}{\partial \alpha}[f(\tau) g(0)]=0$

$\left.\frac{\partial g}{\partial \alpha}\right|_{\alpha=0}=0$

Substituting Eq. (54) into Eq. (50):

$g \frac{\partial f}{\partial \tau}=\frac{f}{\alpha^{2}} \frac{\partial}{\partial \alpha}\left(\alpha^{2} \frac{\partial g}{\partial \alpha}\right)-\kappa^{2} f g$

$\frac{1}{f} \frac{\partial f}{\partial \tau}=\frac{1}{g \alpha^{2}} \frac{\partial}{\partial \alpha}\left(\alpha^{2} \frac{\partial g}{\partial \alpha}\right)-\kappa^{2}$

$\frac{1}{f} \frac{\partial f}{\partial \tau}=C=\frac{1}{g \alpha^{2}} \frac{\partial}{\partial \alpha}\left(\alpha^{2} \frac{\partial g}{\partial \alpha}\right)-\kappa^{2}$

$\frac{1}{f} \frac{d f}{d \tau}=C$

which has general solution:

$f=C_{1} e^{C \tau}$

and:

$\frac{1}{g \alpha^{2}} \frac{d}{d \alpha}\left(\alpha^{2} \frac{d g}{d \alpha}\right)-\kappa^{2}=C$ 


$$
\begin{aligned}
& \frac{1}{\alpha^{2}} \frac{d}{d \alpha}\left(\alpha^{2} \frac{d g}{d \alpha}\right)-\left(C+\kappa^{2}\right) g=0 \\
& \frac{1}{\alpha^{2}} \frac{d}{d \alpha}\left(\alpha^{2} \frac{d g}{d \alpha}\right)+D^{2} g=0
\end{aligned}
$$

in which:

$D^{2} \equiv-C-\kappa^{2}$

which has general solution (using Eq. (C.1-6a) of [10]):

$g=\frac{C_{2}}{\alpha} \cos D \alpha+\frac{C_{3}}{\alpha} \sin D \alpha$

Subjecting Eq. (68) to the rim condition, Eq. (56):

$0=C_{2} \cos D+C_{3} \sin D$

Differentiating Eq. (68):

$\frac{d g}{d \alpha}=\frac{-C_{2} \alpha D \sin D \alpha+C_{3} \alpha \cos D \alpha-C_{2} \cos D \alpha-C_{3} \sin D \alpha}{\alpha^{2}}$

and subjecting this to Eq. (58):

$0=C_{2}$

and thus:

$g=\frac{C_{3}}{\alpha} \sin D \alpha$

and our rim condition, Eq. (56), gives:

$0=C_{3} \sin D$

so that:

$D=n \pi$

where, following EXAMPLE 4.1-2 of [10]:

$n=0, \pm 1, \pm 2, \ldots$

and thus [from definition Eq. (67)]:

$C=-n^{2} \pi^{2}-\kappa^{2}$

Thus, following EXAMPLE 12.1-2 of [10]:

$g_{n}=\frac{C_{3}}{\alpha} \sin n \pi \alpha$

Substituting Eqs. (63), (76) and (77) into Eq. (54) yields:

$\mathrm{X}=\sum_{n=-\infty}^{\infty} \frac{\mathrm{A}_{n}}{\alpha} e^{-\left(n^{2} \pi^{2}+\kappa^{2}\right) \tau} \sin n \pi \alpha$

$\mathrm{X}=\sum_{n=1}^{\infty} \frac{A_{n}}{\alpha} e^{-\left(n^{2} \pi^{2}+\kappa^{2}\right) \tau} \sin n \pi \alpha$

Applying Eq. (52) to Eq. (79)(b):

$\mathrm{X}(\alpha, 0)=1=\sum_{n=1}^{\infty} \frac{A_{n}}{\alpha} \sin n \pi \alpha$

so that:

$\alpha=\sum_{n=1}^{\infty} A_{n} \sin n \pi \alpha$ 
and solving for $A_{n}$ :

$$
\begin{aligned}
& \int_{0}^{1} \alpha \sin m \pi \alpha d \alpha=\sum_{n=1}^{\infty} A_{n} \int_{0}^{1} \sin n \pi \alpha \sin m \pi \alpha d \alpha \\
& \frac{\sin m \pi-m \pi \cos m \pi}{m^{2} \pi^{2}}=A_{m} \frac{m \pi-\cos m \pi \sin m \pi}{2 m \pi} \\
& \frac{-2(-1)^{m}}{m \pi}=A_{m}
\end{aligned}
$$

and thus:

$$
A_{n}=\frac{-2(-1)^{n}}{n \pi}
$$

Substituting into Eq. (79):

$$
\mathrm{X} \equiv \frac{\mathcal{C}_{A}}{\mathcal{C}_{A 0}}=-2 \sum_{n=1}^{\infty} \frac{(-1)^{n}}{n \pi \alpha} e^{-\left(n^{2} \pi^{2}+\kappa^{2}\right) \tau} \sin n \pi \alpha
$$

which is the dimensionless concentration profile for time-dependent diffusion between a cone and a plate with first order reaction and is illustrated in Figure 4 through Figure 6 for low, medium and high reaction rates, respectively.

\section{a. Total Oxidation}

Substituting Eq. (86) into Eq. (5) yields:

$$
\begin{aligned}
& \mathrm{d}=-2 c_{A 0} k^{\prime \prime \prime} \int_{0}^{t} \sum_{n=1}^{\infty} \frac{(-1)^{n}}{n \pi \alpha} e^{-\left(n^{2} \pi^{2}+\kappa^{2}\right) \tau} \sin n \pi \alpha d t \\
& \frac{\mathrm{d}}{c_{A 0}}=-2 \frac{k^{\prime \prime \prime} R^{2}}{\mathcal{D}_{A B}} \int_{0}^{t} \sum_{n=1}^{\infty} \frac{(-1)^{n}}{n \pi \alpha} e^{-\left(n^{2} \pi^{2}+\kappa^{2}\right) \tau} \sin n \pi \alpha d\left(\frac{t \mathcal{O}_{A B}}{R^{2}}\right) \\
& \frac{\mathrm{d}}{c_{A 0}}=-2 \kappa^{2} \sum_{n=1}^{\infty} \frac{(-1)^{n} \sin n \pi \alpha}{n \pi \alpha} \int_{0}^{\tau} e^{-\left(n^{2} \pi^{2}+\kappa^{2}\right) \tau} d \tau \\
& \frac{\mathrm{d}}{\kappa^{2} C_{A 0}}=-2 \sum_{n=1}^{\infty} \frac{(-1)^{n} \sin n \pi \alpha}{n \pi \alpha}\left[\frac{1-e^{-\left(n^{2} \pi^{2}+\kappa^{2}\right) \tau}}{n^{2} \pi^{2}+\kappa^{2}}\right]
\end{aligned}
$$

which is the concentration profile of degradate when the sample is blanketed with nitrogen. By degradate, we mean the amount of oxygen consumed by the polymer oxidation.

\section{UNSTEADY DEGRADATION WITHOUT NITROGEN BLANKETING}

Now suppose that the rim concentration of oxygen was not zero, so that:

$c_{A}(R, t)=c_{A R}$

for which the steady state solution is given by Eqs. (29). We need to combine the transient part of the nitrogen blanketing problem, Eq. (86), with the steady state part of the air bath problem, Eq. (29), as follows: 
$\chi(\alpha, \tau) \equiv \frac{c_{A}(\alpha, \tau)}{c_{A R}}=\chi_{s}(\alpha)+\chi_{t}(\alpha, \tau)$

To get the transient part, we revise the atmospheric boundary condition, Eq. (34), to: $c_{A}(R, t)=c_{A R}$

so that Eq. (51) becomes:

$\chi(1, \tau)=\chi_{s}(1)+\chi_{t}(1, \tau)=1$

and since from Eq. (29):

$\chi_{s}(1)=1$

we rearrange Eq. (94) to:

$\chi_{t}(1, \tau)=0$

The initial condition must also be revised to:

$\chi(\alpha, 0)=\chi_{s}(\alpha)+\chi_{t}(\alpha, 0)=\frac{c_{A 0}}{c_{A R}} \equiv \chi_{0}$

so that:

$\chi_{t}(\alpha, 0)=\chi_{0}-\frac{1}{\alpha} \frac{\sinh \kappa \alpha}{\sinh \kappa}$

Because the initial condition is the only difference from the nitrogen blanket, we can start with Eq. (79):

$\chi_{t}=\sum_{n=1}^{\infty} \frac{B_{n}}{\alpha} e^{-\left(n^{2} \pi^{2}+\kappa^{2}\right) \tau} \sin n \pi \alpha$

which we subject to Eq. (98) to get:

$\chi_{0} \alpha-\frac{\sinh \kappa \alpha}{\sinh \kappa}=\sum_{n=1}^{\infty} B_{n} \sin n \pi \alpha$

Solving for $B_{n}$, we get:

$\int_{0}^{1}\left(\chi_{0} \alpha-\frac{\sinh \kappa \alpha}{\sinh \kappa}\right) \sin m \pi \alpha d \alpha=\int_{0}^{1} \sum_{n=1}^{\infty} B_{n} \sin n \pi \alpha \sin m \pi \alpha d \alpha$

$\frac{\chi_{0}(\sin m \pi-m \pi \cos m \pi)}{m^{2} \pi^{2}}+\frac{m \pi(\cosh 2 \kappa+\sinh 2 \kappa-1) \cos m \pi-\kappa \sin m \pi(\sinh 2 \kappa+\cosh 2 \kappa+1)}{\left(m^{2} \pi^{2}+\kappa^{2}\right)(\cosh 2 \kappa+\sinh 2 \kappa-1)}=B_{m} \frac{m \pi-\cos m \pi \sin m \pi}{2 m \pi}$

$B_{n}=2(-1)^{n}\left(\frac{n \pi}{n^{2} \pi^{2}+\kappa^{2}}-\frac{\chi_{0}}{n \pi}\right)$

which gives us:

$\chi(\alpha, \tau)=\frac{1}{\alpha} \frac{\sinh \kappa \alpha}{\sinh \kappa}+\sum_{n=1}^{\infty} \frac{2(-1)^{n}}{\alpha}\left(\frac{n \pi}{n^{2} \pi^{2}+\kappa^{2}}-\frac{\chi_{0}}{n \pi}\right) e^{-\left(n^{2} \pi^{2}+\kappa^{2}\right) \tau} \sin n \pi \alpha$

where the reactivity to diffusivity ratio, $\kappa$, and the initial to rim oxygen concentration ratio, $\chi_{0}$, are the only adjustable parameters. Figure 7 illustrates the concentration profile of oxygen when the initial and rim concentrations match, $\chi_{0}=1$.

Eq. (104) can be normalized using $\chi_{0}$ to find:

$\mathrm{X}(\alpha, \tau) \equiv \frac{\chi(\alpha, \tau)}{\chi_{0}}=\frac{1}{\chi_{0} \alpha} \frac{\sinh \kappa \alpha}{\sinh \kappa}+\sum_{n=1}^{\infty} \frac{2(-1)^{n}}{\chi_{0} \alpha}\left(\frac{n \pi}{n^{2} \pi^{2}+\kappa^{2}}-\frac{\chi_{0}}{n \pi}\right) e^{-\left(n^{2} \pi^{2}+\kappa^{2}\right) \tau} \sin n \pi \alpha$ 
For the special case initially devoid of oxygen, $\chi_{0}=0$, we must use Eq. (104). By contrast, if a sample equilibrates at room temperature, and is then inserted into the hot cone-plate fixture for study at elevated temperature, $c_{A 0}>c_{A R}$, then Eq. (105) is called for (see Figure 8). If the sample is degassed before testing, so that its initial concentration of oxygen falls below the rim concentration, $c_{A 0}<c_{A R}$, then Eq. (104) is appropriate (see Figure 9). In normal laboratory practice, we see $c_{A 0}>c_{A R}$ more often than $c_{A 0}<c_{A R}$.

\section{a. Total Oxidation}

Substituting Eq. (104) into Eq. (5) gives:

$$
\begin{aligned}
& \mathrm{d}=k^{\prime \prime \prime} C_{A R} \int_{0}^{t} \frac{1}{\alpha} \frac{\sinh \kappa \alpha}{\sinh \kappa}+\sum_{n=1}^{\infty} \frac{2(-1)^{n}}{\alpha}\left(\frac{n \pi}{\left(n^{2} \pi^{2}+\kappa^{2}\right)}-\frac{\chi_{0}}{n \pi}\right) e^{-\left(n^{2} \pi^{2}+\kappa^{2}\right) \tau} \sin n \pi \alpha d t \\
& \mathrm{~d}=k^{\prime \prime \prime} C_{A R}\left(\frac{1}{\alpha} \frac{\sinh \kappa \alpha}{\sinh \kappa} t+\sum_{n=1}^{\infty} \frac{2(-1)^{n} R^{2}}{\alpha \mathscr{D}}\left(\frac{1-e^{-\left(n^{2} \pi^{2}+\kappa^{2}\right) \tau}}{n^{2} \pi^{2}+\kappa^{2}}\right)\left(\frac{n \pi}{\left(n^{2} \pi^{2}+\kappa^{2}\right)}-\frac{\chi_{0}}{n \pi}\right) \sin n \pi \alpha\right) \\
& \frac{\mathrm{d}}{c_{A 0}}=\frac{\kappa^{2}}{\chi_{0} \alpha}\left(\frac{\sinh \kappa \alpha}{\sinh \kappa} \tau+\sum_{n=1}^{\infty} 2(-1)^{n}\left(\frac{1-e^{-\left(n^{2} \pi^{2}+\kappa^{2}\right) \tau}}{n^{2} \pi^{2}+\kappa^{2}}\right)\left(\frac{n \pi}{n^{2} \pi^{2}+\kappa^{2}}-\frac{\chi_{0}}{n \pi}\right) \sin n \pi \alpha\right)
\end{aligned}
$$

which is the concentration profile of degradate when the sample edge is exposed to oxygen.

\section{WORKED EXAMPLE: NITROGEN BLANKETING}

A rheologist wants to know if she needs to use a nitrogen blanket when measuring the viscoelastic properties of her polymer melt undergoing steady shear in a coneplate rheometer. Specifically, she needs to know what the critical dimensionless time, $\tau_{c}$, at which the degradation in a sample exposed to air greatly exceeds that of a sample subjected to a nitrogen blanket. At the temperature of her experiment, she knows the diffusivity of oxygen in her polymer is $\mathscr{O}_{A B}=1.10 \times 10^{-9} \mathrm{~m}^{2} / \mathrm{s}$. She also knows that the rate of oxidative degradation is first order in polymer concentration with a rate coefficient of $k^{\prime \prime \prime}=1.70 \times 10^{-4} \mathrm{~s}^{-1}$. The cone and plate radii are $R=1.0 \mathrm{~cm}$.

Because she is interested in the total degradation, she compares Eqs. (89) and (108) by plotting the degradate concentration profiles versus dimensionless time in Figure 10. From this figure, the engineer sees that degradate concentration for a sample exposed to oxygen immediately diverges from that of the sample blanketed in nitrogen at $\tau=0$. However, near the cone apex the amount of degradation with and without nitrogen blanketing is similar for short times. Subtracting Eq. (108) from Eq. (89) and dividing by Eq. (89) yields the percent difference in degradate concentration from the nitrogen blanketed case. If the engineer can tolerate a 5\% increase in degradate concentration for her sample exposed to oxygen, she finds that the critical runtime for her measurements at $\alpha=[1,0.5,0.001]$ are $\tau_{c}=[0,0.04,0.09]$, which 
corresponds to $t=[0,3600,8200] \mathrm{s}$. Thus, she can run her sample for an hour before the degradation exceeds her tolerance at the point halfway between the cone tip and the outer edge. Since the material at the sample edge has the largest impact on her measurements, she finds that using a nitrogen blanket always reduces degradation and improves her measurement accuracy. She next explores the ability of cone-plate partitioning (without nitrogen blanketing) to reduce the effect of degradation on her measurements.

\section{WORKED EXAMPLE: CONE-PLATE PARTITIONING}

The polymer engineer is still studying the polymer in section $\mathrm{V}$. She wants to know if using a cone-partitioned-plate will increase the amount of time she can run her experiment without a nitrogen blanket. She can still only tolerate a $5 \%$ increase in degradate concentration for her sample exposed to oxygen. She begins by plotting, in Figure 11, degradate concentration versus radius for exposure to air and to nitrogen. From Figure 11, she notices that for short times the degradation profiles only differ near the sample edge and that the maximum degradation occurs near the center for nitrogen-blanketed samples and near the edge for samples exposed to air. Using a similar method to the previous section, the engineer determines the critical radii at which the degradation in her sample exposed to oxygen exceeds $5 \%$ of the degradation in the nitrogen-blanketed sample. She finds that for $\tau=[0,0.01,0.05,0.1,0.5]$, or $t=[0,0.25,1.26,2.53,12.6] \mathrm{h}$, the critical radii are $\alpha_{c}=[1,0.77,0.44,0,0]$, which translate to $r_{c}=[10,7.7,4.4,0,0] \mathrm{mm}$ for a cone having radius $R=10 \mathrm{~mm}$. Thus, she can operate without a nitrogen blanket for 1.26 hours using a partitioned-plate have inner partition radius of $R_{p}=4.4 \mathrm{~mm}$.

Commercial cone-plate partitioned rheometers typically employ an inner radius of $\frac{1}{4} \leq \alpha_{p} \leq \frac{1}{2}$, [8,11]. If she uses $\alpha_{p}=0.32$, she can run her measurement for 1.39 hours without degradation impacting her measurements. This is a distinct improvement over the partitionless cone-plate of the previous example, where without a nitrogen blanket, degradation impacts measurement immediately. Thus, nitrogen blanketing is called for in a cone-plate when the polymer degrades readily. However, conepartitioned-plates can be the better solution to degradation if the measurement duration does not exceed the critical time for the inner partition radius.

\section{UNSTEADY OXIDATION WITH ANTIOXIDANT DOPING}

To avoid sample oxidation, the polymer is commonly doped with antioxidant. The uninitiated may expect antioxidant doping to suppress all polymer degradation. In this section, we test this expectation and investigate the nature of the polymer degradation induction time arising from adding antioxidant. Antioxidant consumption follows a reaction that is zeroth order in antioxidant concentration [12], $c_{N}$, and thus:

$R_{N}=-k_{N}^{\prime \prime \prime} c_{A}$

where we use first order dependence on oxygen concentration as the simplest relevant case. 
To incorporate the effects of antioxidants, we modify the reaction term in Eq. (38) to include both Eq. (4) and Eq. (109) to get:

$c \frac{\partial x_{A}}{\partial t}=\frac{-1}{r^{2}} \frac{\partial}{\partial r}\left(r^{2} J_{A r}^{*}\right)-\left(k^{\prime \prime \prime}+k_{N}^{\prime \prime \prime}\right) c_{A}$

which in turn modifies only the dimensionless rate constant (see EXAMPLE 18.3-1 of [10]; [13]):

$\mathrm{Da}_{\mathrm{II}}=\kappa_{\mathrm{NA}}^{2} \equiv \frac{\left(k^{\prime \prime \prime}+k_{N}^{\prime \prime \prime}\right) R^{2}}{\mathscr{D}_{A B}}$

Eqs. (110) and (38) share the same form, and if $\kappa$ is replaced with $\kappa_{N A}$, any solution to Eq. (38) can be taken over for antioxidant doping. Thus, so long as the antioxidant has not yet been exhausted at the edge, Eqs. (86) (with nitrogen blanketing) or (105) (without nitrogen blanketing) can be taken over for antioxidant doping.

Focusing on the latter, an antioxidant-doped sample without nitrogen blanketing, we can determine a new degradation profile as well as an antioxidant concentration profile. We begin with the latter using the definition:

$c_{N} \equiv c_{N 0}+\int_{0}^{t} R_{N} d t^{\prime}=c_{N 0}-k_{N}^{\prime \prime \prime} \int_{0}^{t} c_{A} d t^{\prime}$

Inserting Eq. (105) into Eq. (112), we find:

$\left.c_{N}=c_{N 0}-k_{N}^{\prime \prime \prime} \int_{0}^{t} \frac{c_{A R}}{\alpha} \frac{\sinh \kappa_{N A} \alpha}{\sinh \kappa_{N A}}+c_{A R} \sum_{n=1}^{\infty} \frac{2(-1)^{n}}{\alpha}\left(\frac{n \pi}{n^{2} \pi^{2}+\kappa_{N A}^{2}}-\frac{\chi_{0}}{n \pi}\right) e^{-\left(n^{2} \pi^{2}+\kappa_{N A}^{2}\right) \tau} \sin n \pi \alpha d t^{\prime}(1)\right)$
$c_{N}=c_{N 0}-k_{N}^{\prime \prime \prime} c_{A R}\left(\frac{1}{\alpha} \frac{\sinh \kappa_{N A} \alpha}{\sinh \kappa_{N A}} t+\sum_{n=1}^{\infty} \frac{2(-1)^{n} R^{2}}{\alpha \mathscr{D}_{A B}}\left(\frac{1-e^{-\left(n^{2} \pi^{2}+\kappa_{N A}^{2}\right) \tau}}{n^{2} \pi^{2}+\kappa_{N A}^{2}}\right)\left(\frac{n \pi}{\left(n^{2} \pi^{2}+\kappa_{N A}^{2}\right)}-\frac{\chi_{0}}{n \pi}\right) \sin n \pi \alpha\right)$
$\mathrm{X}_{N} \equiv \frac{c_{N}}{c_{N 0}}=1-\frac{k_{N}^{\prime \prime \prime}}{\chi_{N 0}}\left(\frac{1}{\alpha} \frac{\sinh \kappa_{N A} \alpha}{\sinh \kappa_{N A}} t+\sum_{n=1}^{\infty} \frac{2(-1)^{n} R^{2}}{\alpha \mathscr{D}_{A B}}\left(\frac{1-e^{-\left(n^{2} \pi^{2}+\kappa_{N A}^{2}\right) \tau}}{n^{2} \pi^{2}+\kappa_{N A}^{2}}\right)\left(\frac{n \pi}{\left(n^{2} \pi^{2}+\kappa_{N A}^{2}\right)}-\frac{\chi_{0}}{n \pi}\right) \sin n \pi \alpha\right)$
$\mathrm{X}_{N} \equiv \frac{c_{N}}{c_{N 0}}=1-\frac{\kappa_{N}^{2}}{\chi_{N 0} \alpha}\left(\frac{\sinh \kappa_{N A} \alpha}{\sinh \kappa_{N A}} \tau+\sum_{n=1}^{\infty} 2(-1)^{n}\left(\frac{1-e^{-\left(n^{2} \pi^{2}+\kappa_{N A}^{2}\right) \tau}}{n^{2} \pi^{2}+\kappa_{N A}^{2}}\right)\left(\frac{n \pi}{n^{2} \pi^{2}+\kappa_{N A}^{2}}-\frac{\chi_{0}}{n \pi}\right) \sin n \pi \alpha\right)$

where $\kappa_{N}^{2} \equiv k_{N}^{\prime \prime \prime} R^{2} / \mathscr{D}_{A B}$ and $\chi_{N 0} \equiv c_{N 0} / c_{A R}$. From Eq. (116), we get the induction time at the edge, that is, the time required for the antioxidant to vanish. When $\chi_{\mathrm{N} 0}=10$ and $k_{N}^{\prime \prime \prime}=10 k^{\prime \prime \prime}$, we find that the antioxidant vanishes at the edge in 1.64 hours, and that the degradate concentration exceeds $5 \%$ of the oxygen concentration at the sample edge in 49.4 minutes. Increasing $\chi_{\mathrm{N} 0}$ extends the induction time proportionally. This also shows that the presence of antioxidant does not prevent degradation at the rim. However, antioxidants do decelerate degradation by inducing the 49.4 minute induction.

Though Eq. (116) is useful for short times, we must modify Eq. (105) to study the longer term benefit of antioxidants. Specifically, we must account for the absence of antioxidant once it is consumed entirely, at each polymer layer. For simplicity, we use a pseudo-steady state approach for determining the radius of the receding antioxidant front. First, we find the steady state solutions for the regions without: 
$\chi_{A} \equiv \frac{c_{A}}{c_{A R}}=\frac{\left(\begin{array}{l}\kappa \sinh \kappa_{N A} \alpha_{I}\left(\sinh \kappa \alpha_{I} \sinh \kappa \alpha-\cosh \kappa \alpha_{I} \cosh \kappa \alpha\right) \\ +\kappa_{N A} \cosh \kappa_{N A} \alpha_{I}\left(\sinh \kappa \alpha_{I} \cosh \kappa \alpha-\cosh \kappa \alpha_{I} \sinh \kappa \alpha\right)\end{array}\right)}{\alpha\left(\begin{array}{l}\kappa \sinh \kappa_{N A} \alpha_{I}\left(\sinh \kappa \alpha_{I} \sinh \kappa-\cosh \kappa \alpha_{I} \cosh \kappa\right) \\ +\kappa_{N A} \cosh \kappa_{N A} \alpha_{I}\left(\sinh \kappa \alpha_{I} \cosh \kappa-\cosh \kappa \alpha_{I} \sinh \kappa\right)\end{array}\right)} ; \alpha_{I}<\alpha \leq 1$

and with antioxidant:

$\chi_{A} \equiv \frac{c_{A}}{c_{A R}}=\frac{-\kappa \sinh \kappa_{N A} \alpha}{\alpha\left(\begin{array}{l}\kappa \sinh \kappa_{N A} \alpha_{I}\left(\sinh \kappa \alpha_{I} \sinh \kappa-\cosh \kappa \alpha_{I} \cosh \kappa\right) \\ +\kappa_{N A} \cosh \kappa_{N A} \alpha_{I}\left(\sinh \kappa \alpha_{I} \cosh \kappa-\cosh \kappa \alpha_{I} \sinh \kappa\right)\end{array}\right)} ; 0 \leq \alpha \leq \alpha_{I}$

where $\alpha_{I}$ is the radius of the receding antioxidant front. To determine the timedependence of $\alpha_{I}$, we insert Eq. (118) into Eq. (112) to get:

$$
\begin{gathered}
c_{N}=c_{N 0}-k_{N}^{\prime \prime \prime} \int_{0}^{t} \frac{-\kappa c_{A R} \sinh \kappa_{N A} \alpha}{\alpha\left(\begin{array}{l}
\kappa \sinh \kappa_{N A} \alpha_{I}\left(\sinh \kappa \alpha_{I} \sinh \kappa-\cosh \kappa \alpha_{I} \cosh \kappa\right) \\
+\kappa_{N A} \cosh \kappa_{N A} \alpha_{I}\left(\sinh \kappa \alpha_{I} \cosh \kappa-\cosh \kappa \alpha_{I} \sinh \kappa\right)
\end{array}\right)} d t^{\prime} \\
\mathrm{X}_{N}=1+\frac{\kappa_{N}^{2}}{\chi_{N 0}} \frac{\kappa \sinh \kappa_{N A} \alpha}{\alpha\left(\begin{array}{l}
\kappa \sinh \kappa_{N A} \alpha_{I}\left(\sinh \kappa \alpha_{I} \sinh \kappa-\cosh \kappa \alpha_{I} \cosh \kappa\right) \\
+\kappa_{N A} \cosh \kappa_{N A} \alpha_{I}\left(\sinh \kappa \alpha_{I} \cosh \kappa-\cosh \kappa \alpha_{I} \sinh \kappa\right)
\end{array}\right)} \tau
\end{gathered}
$$

Since $\alpha=\alpha_{I}$ when $\mathrm{X}_{N}=0$, so that:

$$
\tau_{I}=-\frac{\chi_{N 0} \alpha_{I}}{\kappa \kappa_{N}^{2}}\left(\begin{array}{l}
\kappa\left(\sinh \kappa \alpha_{I} \sinh \kappa-\cosh \kappa \alpha_{I} \cosh \kappa\right) \\
+\kappa_{N A} \operatorname{coth} \kappa_{N A} \alpha_{I}\left(\sinh \kappa \alpha_{I} \cosh \kappa-\cosh \kappa \alpha_{I} \sinh \kappa\right)
\end{array}\right)
$$

which is the time required for antioxidant to retreat to a particular radius, $\alpha_{I}$.

We find that the antioxidant is completely consumed at the sample edge in 1.63 hours, and that the degradate concentration exceeds $5 \%$ of the oxygen saturation concentration in 4.93 seconds, when $\chi_{N 0}=10$ and $k_{N}^{\prime \prime \prime}=10 k^{\prime \prime \prime}$. As expected, the pseudosteady state approximation underestimates the induction time at the rim as a worst case, since it assumes that the oxygen concentration peaks immediately. If we consider the inner plate radius of a typical cone-partitioned-plate geometry, $\alpha_{p}=0.32$, we find that it takes 16.3 hours for the antioxidant front to retreat to the plate partition. Additionally, the degradate concentration will compromise cone-partitioned-plate measurement after 49.0 minutes. This falls below, and thus underestimates, the time for degradate to impact measurement using an unsteady solution without antioxidant (of 1.39 hours, see above). Because this pseudo-steady state approximation provides a worst-case underestimation, an engineer could expect to have more than 1.39 hours to run a sample using a cone-partitioned-plate. We illustrate the antioxidant and doublyinflected oxygen concentration profiles for this worked example in Figure 12 when $\alpha_{I}=0.32$.

Comparing this worked example, with its precedent, we learn of the advantage of cone-plate partitioning for delaying or suppressing oxidative degradation in coneplate flow. 


\section{CONCLUSION}

We analyze quantitatively the oxidative degradation of a sample in a cone-plate rheometer, as oxygen diffuses inward, radially, from the free boundary (Eq. (86) with nitrogen blanketing, and Eqs. (104)-(105), without). We examine rheometer error mitigation by means of nitrogen blanketing, and also, of cone-plate partitioning (by comparing worked examples). We arrive at exact analytical expressions for the oxygen concentration (Eq. (86) with nitrogen blanketing, and Eqs. (104)-(105), without), and thus, for the degradation rate. We then integrate these rates over time to get the amount of oxygen reacted as a function of radial position and time in the degrading sample (Eq. (89) with nitrogen blanketing, and Eq. (108), without). To illustrate the usefulness of our analytical expressions, we provide two worked examples investigating the effect of nitrogen blanketing and cone-plate partitioning. We find that, though nitrogen blanketing always produces less degradation, its benefits are limited for short times. Comparing our worked examples, we deepen our understanding of the advantage of cone-plate partitioning for delaying or suppressing oxidative degradation in cone-plate flow.

Additionally, cone-plate partitioning provides a simpler solution than nitrogen blanketing, and allows samples to be run for longer times without degradation compromising measurement, even in samples initially saturated with oxygen. We close by examining the effect of antioxidants. We arrive at expressions for the induction time at the edge [Eq. (116)], for the receding antioxidant front [Eq. (121)], for the associated concentration profiles [Eqs. (117)-(118) and Eq. (120)], and thus, degradation rates.

Though the use of nitrogen blanketing in cone-plate viscometry is widespread, we know of no measurements of polymer oxidative degradation in cone-plate viscometry, or in any other rotational rheometer geometry. Since parallel-disk viscometry is used at least as commonly as cone-plate, analysis in cylindrical coordinates will be equally useful. We leave this for another day.

\section{ACKNOWLEDGEMENT}

This research was undertaken, in part, thanks to support from the Canada Research Chairs program of the Government of Canada for the Natural Sciences and Engineering Research Council of Canada (NSERC) Tier 1 Canada Research Chair in Rheology. This research was also undertaken, in part, thanks to support from the Discovery Grant program of the Natural Sciences and Engineering Research Council of Canada (NSERC) (A.J. Giacomin) and the Vanier Canada Graduate Scholarship (P.H. Gilbert). A.J. Giacomin is indebted to the Faculty of Applied Science and Engineering of Queen's University at Kingston, for its support through a Research Initiation Grant (RIG). P.H. Gilbert is grateful for an International Tuition Award from the School of Graduate Studies of Queen's University at Kingston. 
Table I: Dimensional Variables

\begin{tabular}{|c|c|c|c|}
\hline Name & Symbol & Dimensions & Range \\
\hline $\begin{array}{l}\text { Azimuthal angular spherical } \\
\text { coordinate }\end{array}$ & $\phi$ & $\mathrm{rad}$ & $0<\phi \leq 2 \pi$ \\
\hline Concentration & C & $\mathrm{mol} L^{-3}$ & $\geq 0$ \\
\hline Concentration, antioxidant & $c_{N}$ & $\operatorname{mol} L^{-3}$ & $\geq 0$ \\
\hline $\begin{array}{l}\text { Concentration, initial } \\
\text { antioxidant }\end{array}$ & $c_{N 0}$ & $\operatorname{mol} L^{-3}$ & $\geq 0$ \\
\hline Concentration, initial oxygen & $c_{A 0}$ & $\operatorname{mol} L^{-3}$ & $\geq 0$ \\
\hline Concentration, oxygen & $c_{A}$ & $\mathrm{~mol} L^{-3}$ & $\geq 0$ \\
\hline Concentration, rim oxygen & $c_{A R}$ & $\operatorname{mol} L^{-3}$ & $\geq 0$ \\
\hline Cone angle & $\theta_{c}$ & rad & $0 \leq \theta \leq \pi$ \\
\hline Critical radius & $r_{c}$ & $L$ & $\geq 0$ \\
\hline $\begin{array}{l}\text { Diffusivity of oxygen } \\
\text { through sample }\end{array}$ & $\mathscr{Q}_{A B}$ & $L^{2} t^{-1}$ & $\geq 0$ \\
\hline $\begin{array}{l}\text { First order rate constant, } \\
\text { antioxidant }\end{array}$ & $k_{N}^{\prime \prime \prime}$ & $t^{-1}$ & $\geq 0$ \\
\hline $\begin{array}{l}\text { First order rate constant, } \\
\text { degradate }\end{array}$ & $k^{\prime \prime \prime}$ & $t^{-1}$ & $\geq 0$ \\
\hline $\begin{array}{l}\text { Molar flux of oxygen, } i \text { th } \\
\text { component }\end{array}$ & $J_{A i}^{*}$ & $\operatorname{mol} L^{-2} t^{-1}$ & $\mathbb{R}$ \\
\hline $\begin{array}{l}\text { Polar angular spherical } \\
\text { coordinate }\end{array}$ & $\theta$ & $\mathrm{rad}$ & $0 \leq \theta \leq \pi$ \\
\hline Radial spherical coordinate & $r$ & $L$ & $\geq 0$ \\
\hline Radius, antioxidant interface & $r_{I}$ & $L$ & $\geq 0$ \\
\hline Radius, plate & $R$ & $L$ & $>0$ \\
\hline Radius, plate partition & $R_{p}$ & $L$ & $0<R_{p}<R$ \\
\hline Reaction rate, antioxidant & $R_{N}$ & $\mathrm{~mol} L^{-3} t^{-1}$ & $\leq 0$ \\
\hline Reaction rate, oxygen & $R_{A}$ & $\operatorname{mol} L^{-3} t^{-1}$ & $\leq 0$ \\
\hline Time & $t$ & $t$ & $\mathbb{R}$ \\
\hline Total oxidative degradation & $\mathrm{d}$ & $\operatorname{mol~} L^{-3}$ & $\geq 0$ \\
\hline Velocity, ith component & $v_{i}$ & $L t^{-1}$ & $\mathbb{R}$ \\
\hline
\end{tabular}

Legend: $M \equiv$ mass; $L \equiv$ length; $t \equiv$ time; $T \equiv$ temperature 
Table II: Dimensionless Variables and Groups

\begin{tabular}{|l|c|c|}
\hline \multicolumn{1}{|c|}{ Name } & Symbol & Range \\
\hline Concentration of oxygen & $\chi \equiv c_{A} / c_{A R}$ & $\geq 0$ \\
\hline Concentration of oxygen, steady part & $\chi_{s}$ & $\geq 0$ \\
\hline Concentration of oxygen, transient part & $\chi_{t}$ & $\geq 0$ \\
\hline Mole fraction, oxygen & $x_{A}=c_{A} / c$ & $0 \leq x_{A} \leq 1$ \\
\hline Mole fraction, sample & $x_{B}=c_{B} / c$ & $0 \leq x_{B} \leq 1$ \\
\hline $\begin{array}{l}\text { Normalized concentration of } \\
\text { antioxidant }\end{array}$ & $\mathrm{X}_{N} \equiv c_{N} / c_{N 0}$ & $\geq 0$ \\
\hline Normalized concentration of oxygen & $\mathrm{X} \equiv c_{A} / c_{A 0}$ & $\geq 0$ \\
\hline Radial coordinate & $\alpha \equiv r / R$ & $0 \leq \alpha \leq 1$ \\
\hline Radius, antioxidant interface & $\alpha_{I} \equiv r_{I} / R$ & $0 \leq \alpha_{c} \leq 1$ \\
\hline Radius, critical & $\alpha_{c} \equiv r_{c} / R$ & $0 \leq \alpha_{c} \leq 1$ \\
\hline Radius, partition & $\alpha_{p} \equiv R_{p} / R$ & $0 \leq \alpha_{c} \leq 1$ \\
\hline $\begin{array}{l}\text { Ratio of initial antioxidant to rim } \\
\text { oxygen concentration }\end{array}$ & $\chi_{N 0} \equiv c_{N 0} / c_{A R}$ & $\geq 0$ \\
\hline $\begin{array}{l}\text { Ratio of initial to rim oxygen } \\
\text { concentration }\end{array}$ & $\chi_{0} \equiv c_{A 0} / c_{A R}$ & $\geq 0$ \\
\hline Reaction rate constant, antioxidant & $\kappa_{N}^{2} \equiv k_{N}^{\prime \prime \prime} R^{2} / \mathscr{D}_{A B}$ & $\geq 0$ \\
\hline $\begin{array}{l}\text { Reaction rate constant, combined } \\
\text { degradate and antioxidant }\end{array}$ & $\kappa_{N A}^{2} \equiv\left(k^{\prime \prime \prime}+k_{N}^{\prime \prime \prime}\right) R^{2} / \mathscr{D}_{A B}$ & $\geq 0$ \\
\hline Reaction rate constant, degradate & $\kappa^{2} \equiv k^{\prime \prime \prime} R^{2} / \mathscr{D}_{A B}$ & $\geq 0$ \\
\hline Second Damköhler number & $\mathrm{Da}_{\mathrm{II}}$ & $0 \leq x_{B} \leq 1$ \\
\hline Time & $\tau \equiv t \mathscr{O}_{A B} / R^{2}$ & $>0$ \\
\hline $\begin{array}{l}\text { Time, antioxidant front recession } \\
\text { [Eq. (121)] }\end{array}$ & $\tau_{I}$ & $>0$ \\
\hline Time, critical & $\tau_{c} \equiv t_{c} \mathscr{O}_{A B} / R^{2}$ & $>0$ \\
\hline
\end{tabular}

Legend: $\mathbb{R} \equiv$ all real numbers 


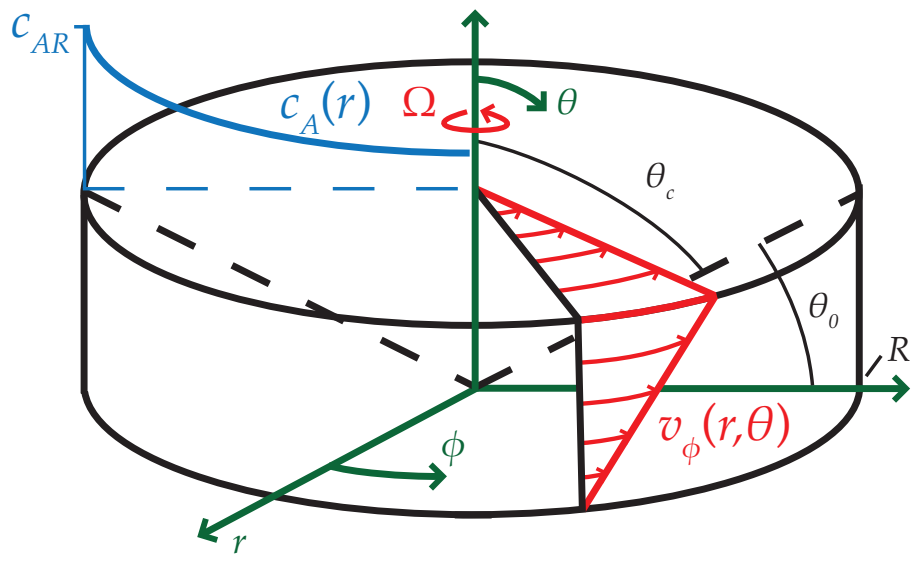

Figure 1: Sketch of sample in cone-plate rheometer without cone-plate partitioning, defining spherical coordinates and boundaries. 


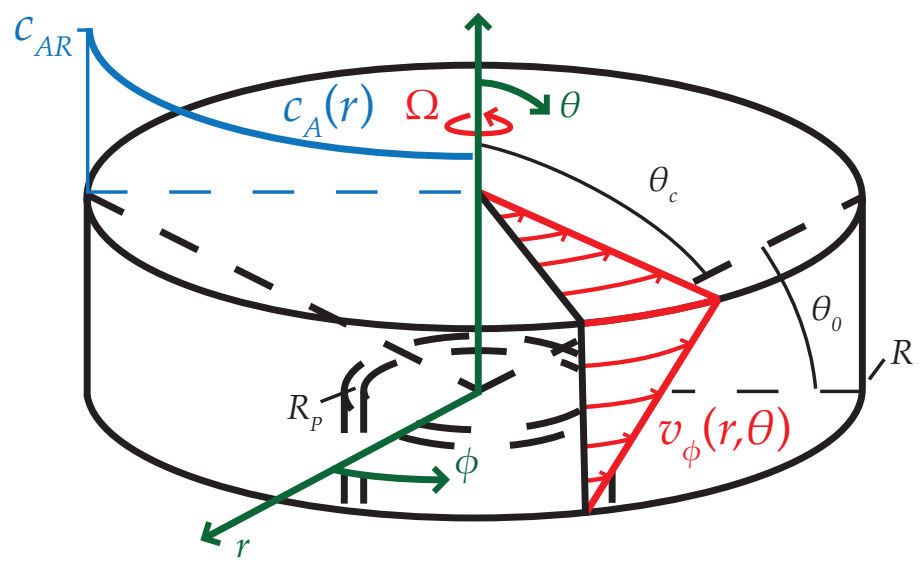

Figure 2: Sketch of sample in cone-plate rheometer with cone-plate partitioning. 


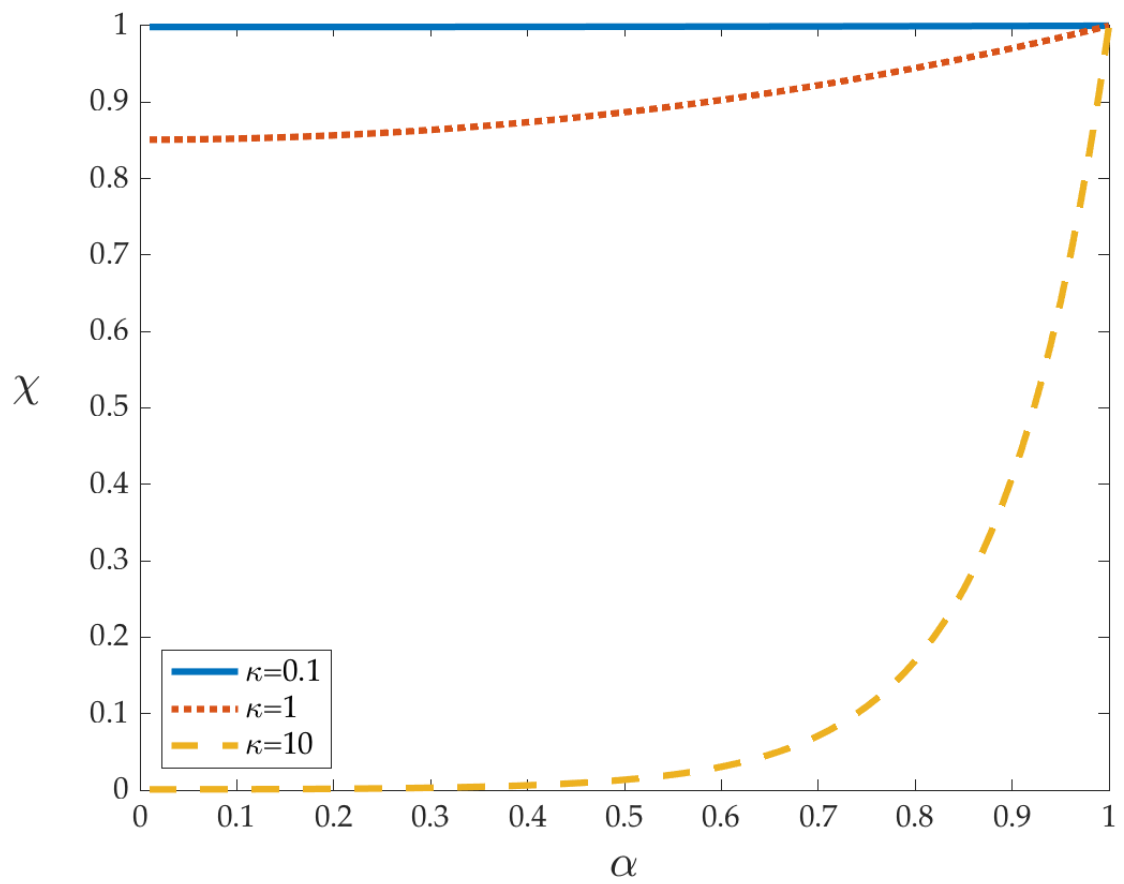

Figure 3: Steady state dimensionless concentration profiles from Eq. (29) for $\kappa=0.1,1,10$. 


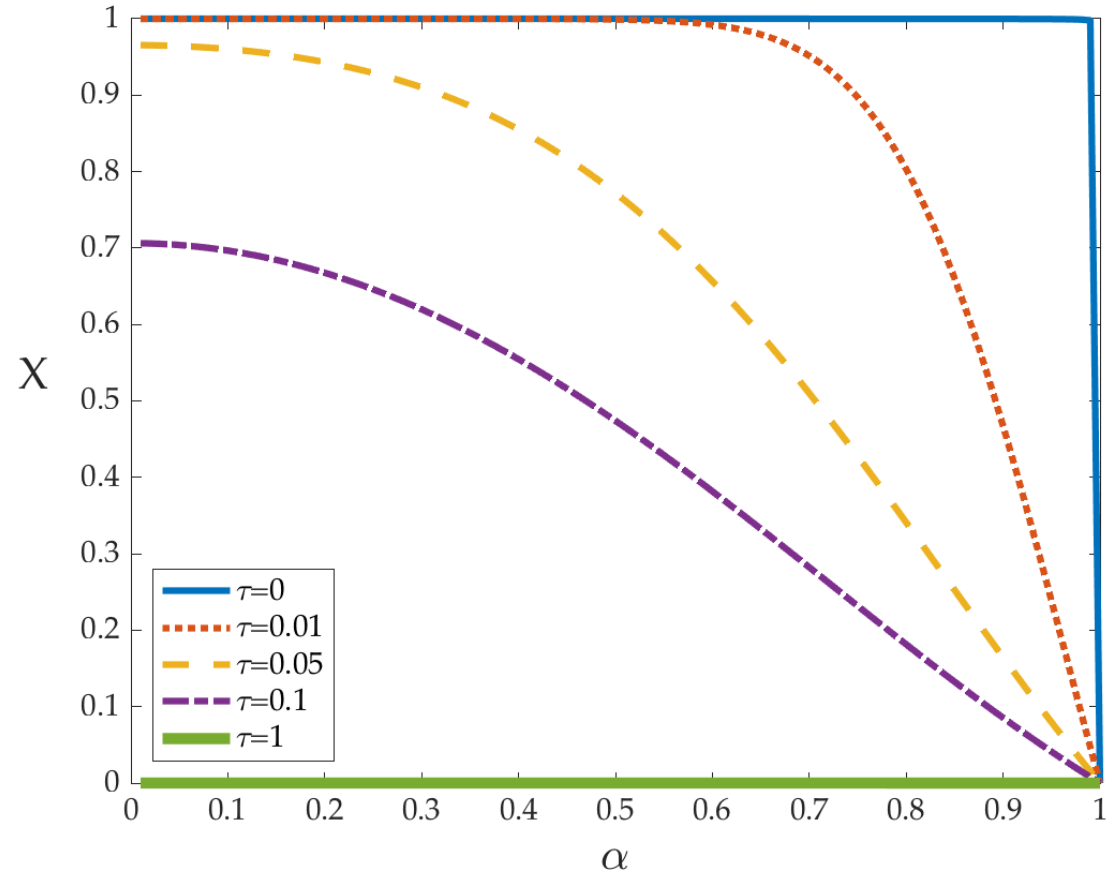

Figure 4: Dimensionless concentration profiles versus radius, from Eq. (86), for five different times with a dimensionless rate constant of $\kappa=0.1$. 


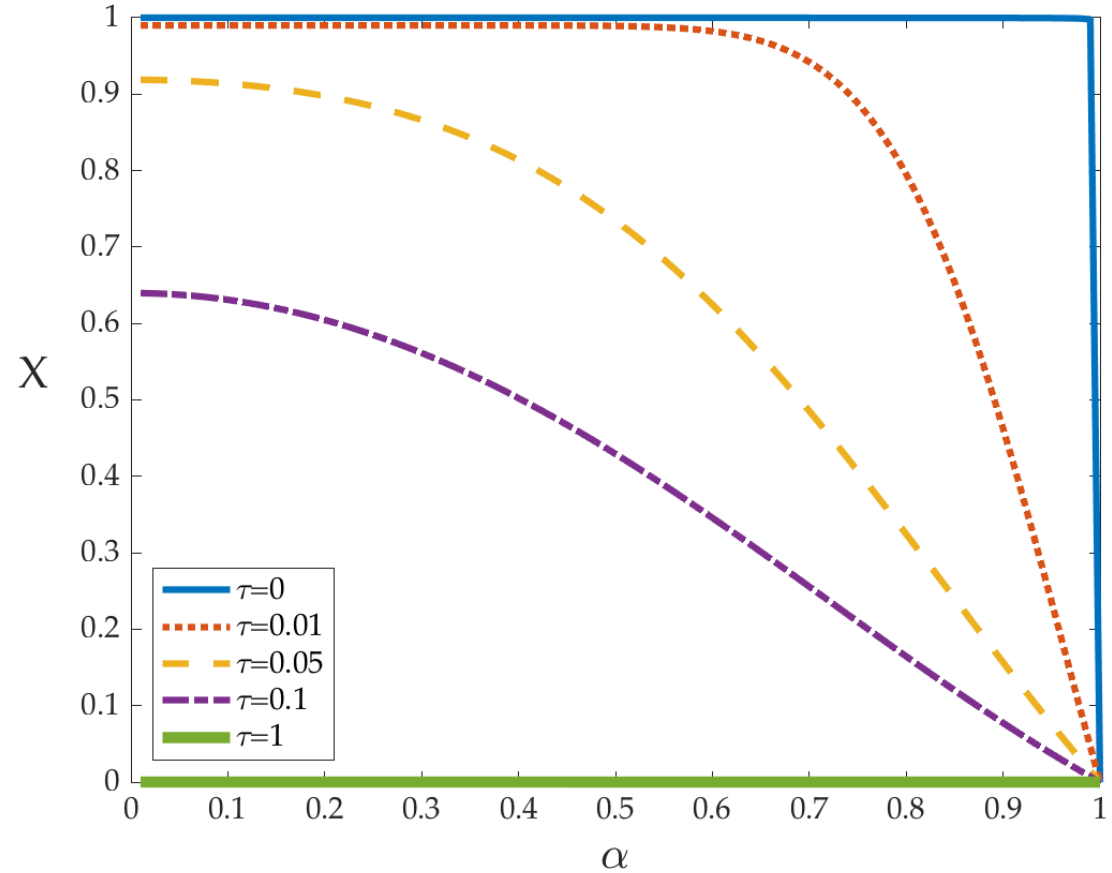

Figure 5: Dimensionless concentration profiles versus radius, from Eq. (86), for five different times with a dimensionless rate constant of $\kappa=1$. 


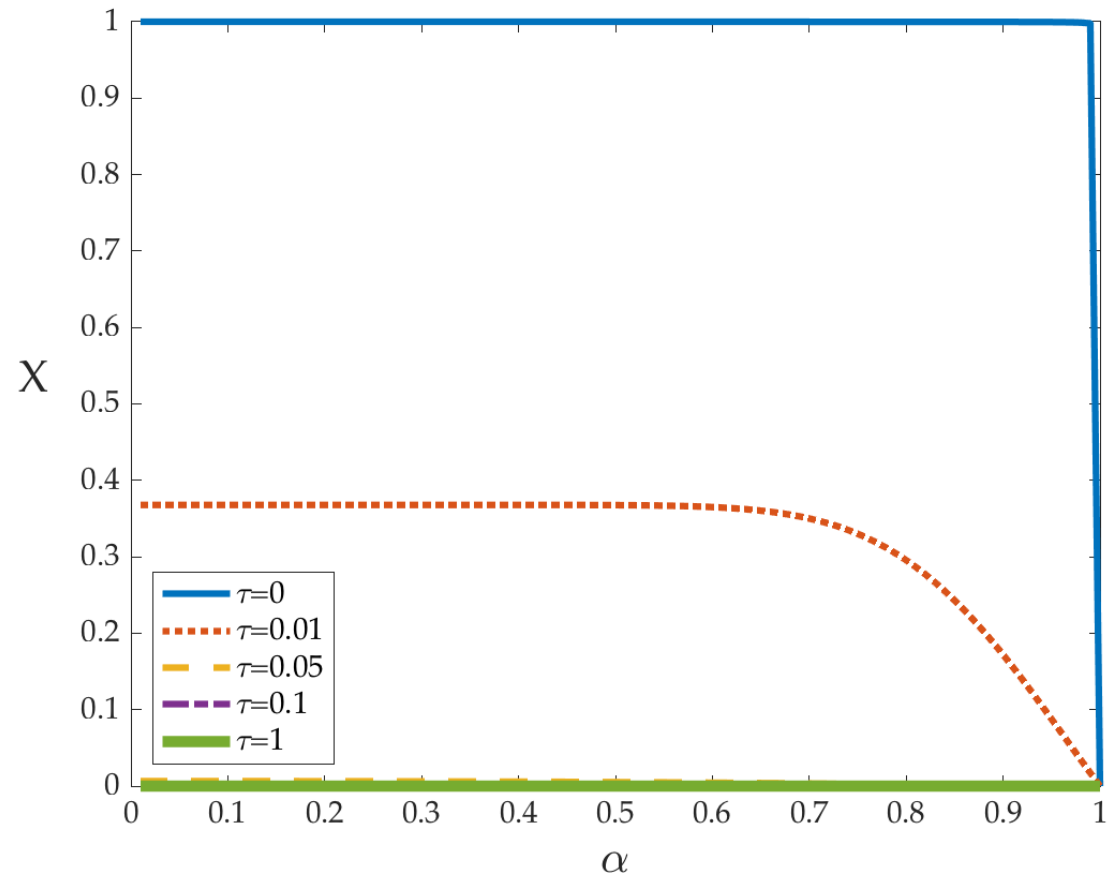

Figure 6: Dimensionless concentration profiles versus radius, from Eq. (86), for five different times with a dimensionless rate constant of $\kappa=10$. 


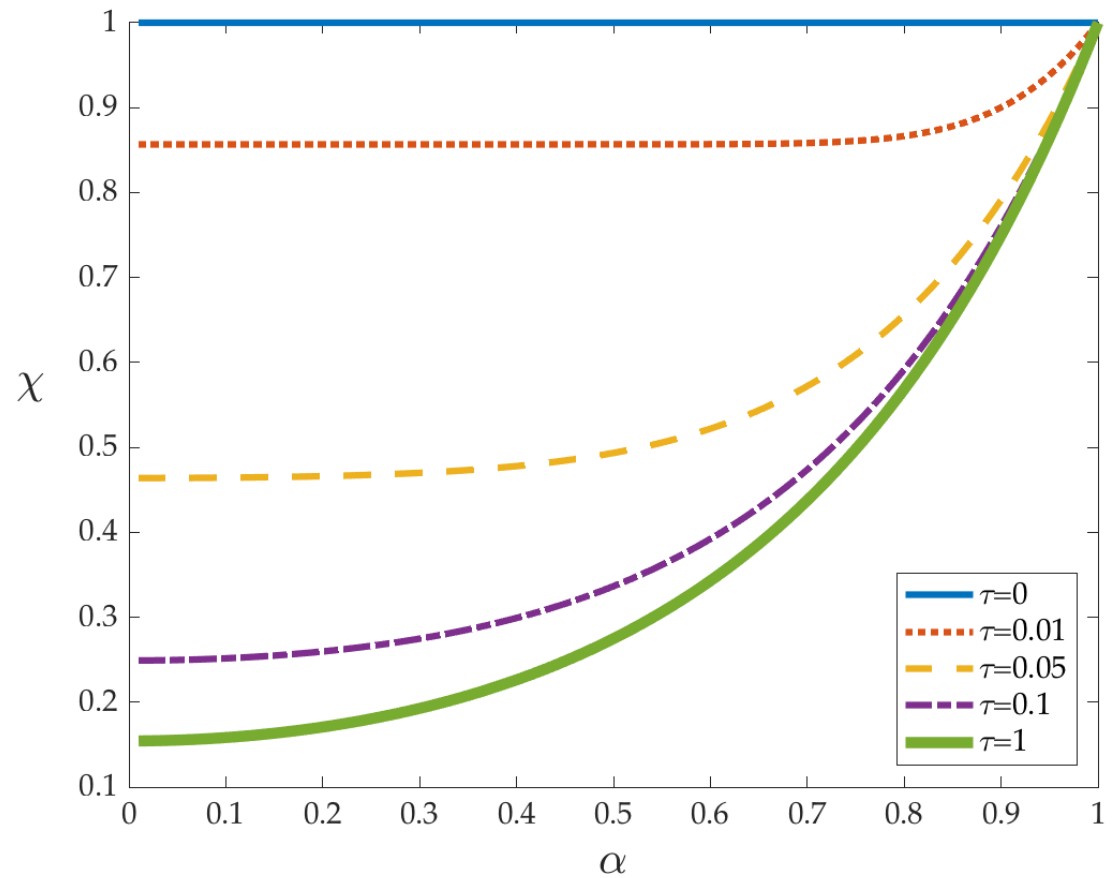

Figure 7: Dimensionless concentration profiles versus radius, from Eq. (104), for five different times with a dimensionless rate constant of $\kappa=3.93$ and an initial to rim oxygen concentration ratio of $\chi_{0}=1$. 


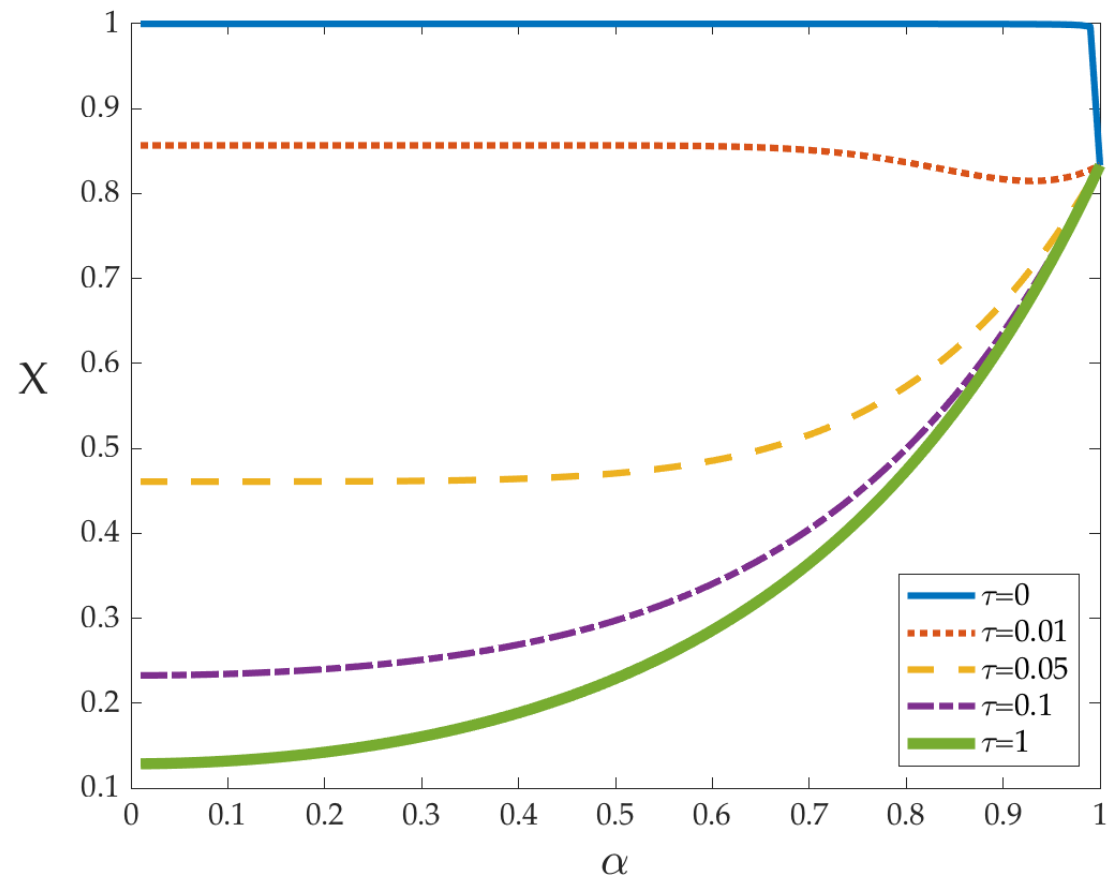

Figure 8: Dimensionless concentration profiles versus radius, from Eq. (105), for five different times with a dimensionless rate constant of $\kappa=3.93$ and an initial to rim oxygen concentration ratio of $\chi_{0}=1.2$. 


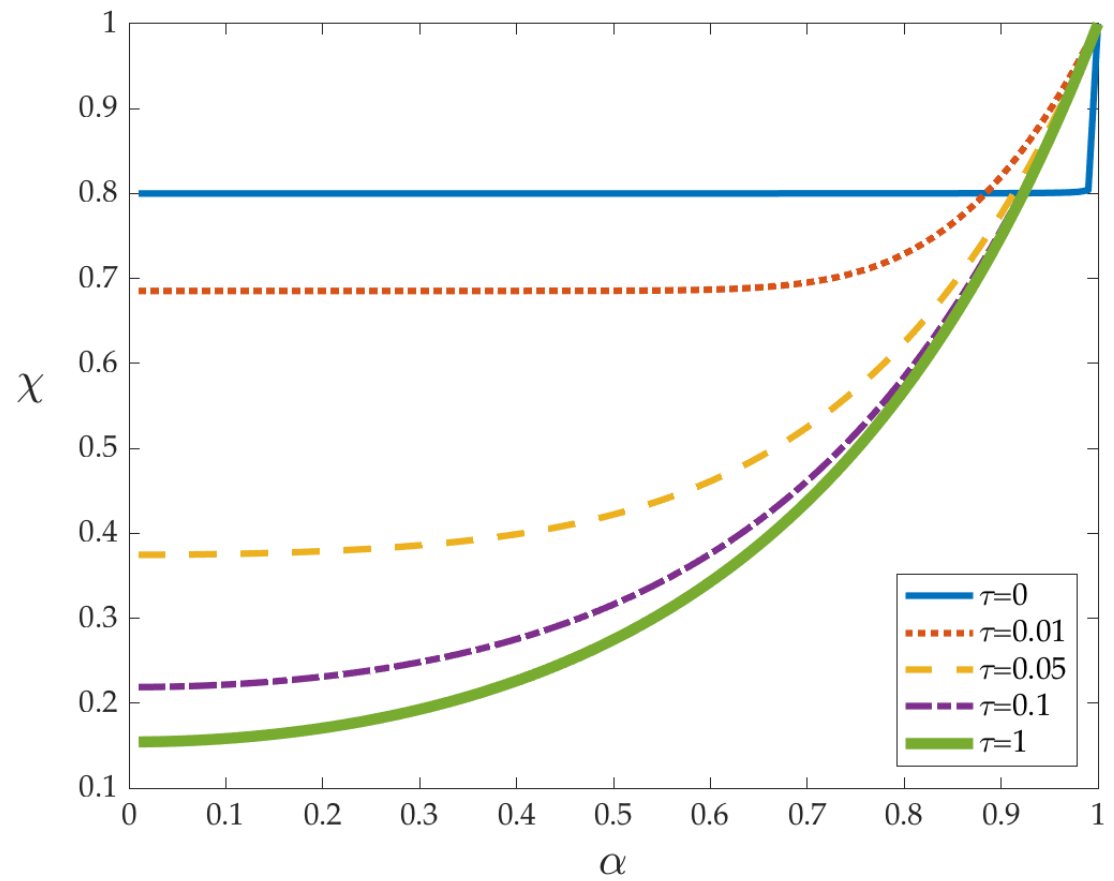

Figure 9: Dimensionless concentration profiles versus radius, from Eq. (104), for five different times with a dimensionless rate constant of $\kappa=3.93$ and an initial to rim oxygen concentration ratio of $\chi_{0}=0.80$. 


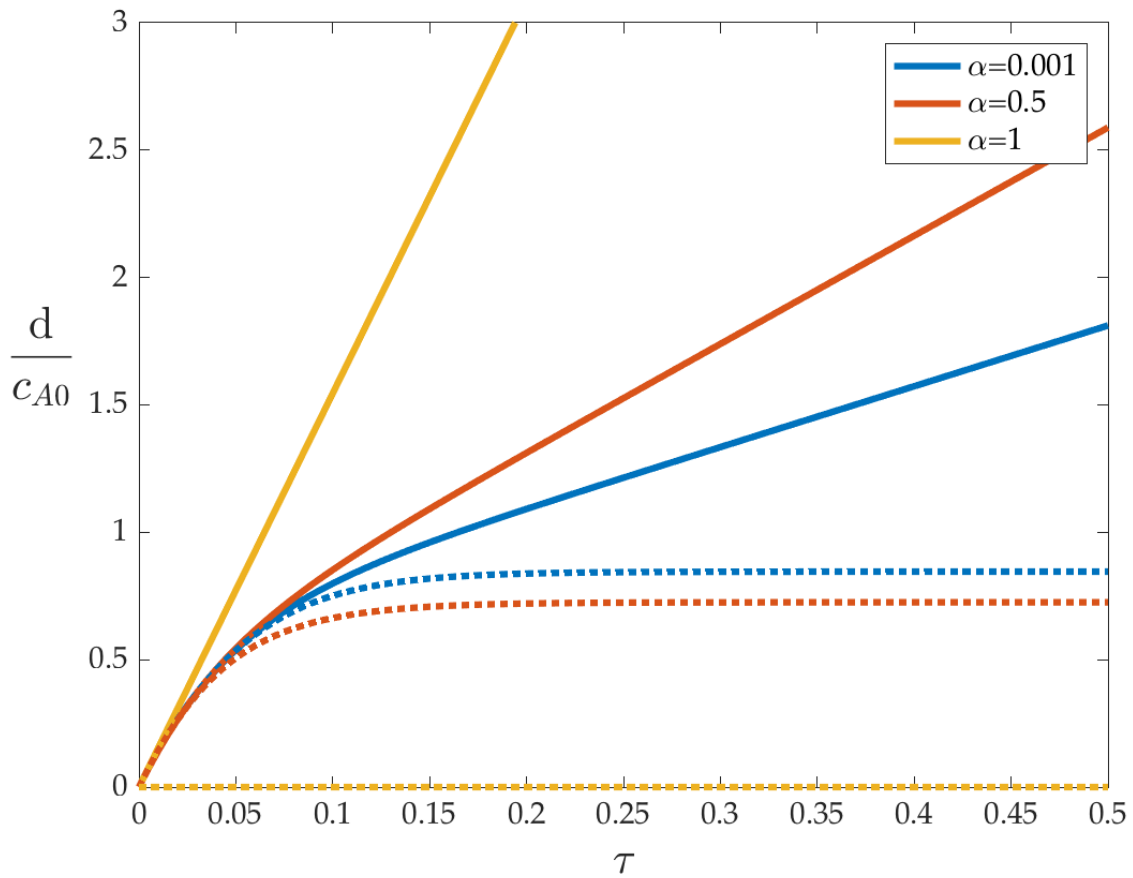

Figure 10: Dimensionless concentration profiles versus time, from Eq. (89) and (108), for three different radii with a dimensionless rate constant of $\kappa=3.93$ and an initial to rim oxygen concentration ratio of $\chi_{0}=1$, where the solid and dashed curves correspond to the sample exposed to oxygen and the sample blanketed with nitrogen. 


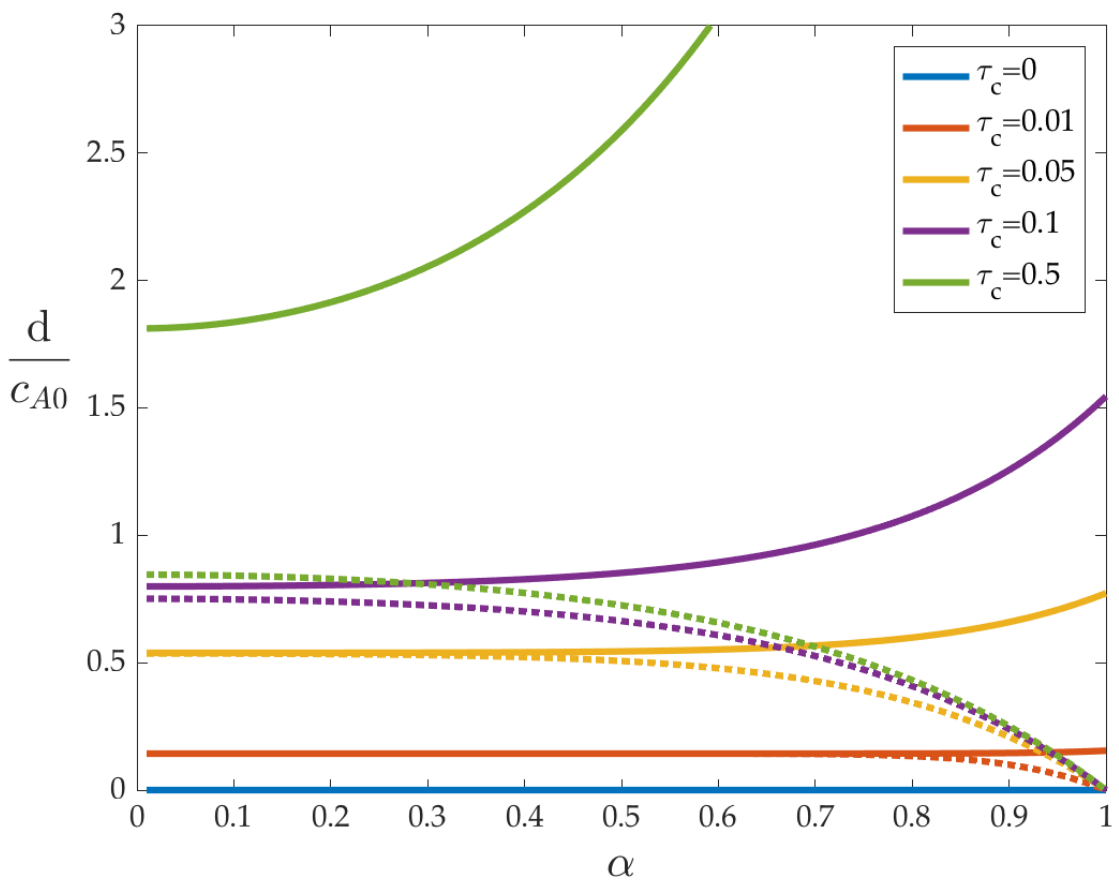

Figure 11: Dimensionless degradate concentration profiles versus radius, from Eq. (89) and (108), for five different times with a dimensionless rate constant of $\kappa=3.93$ and an initial to rim oxygen concentration ratio of $\chi_{0}=1$, where the solid and dashed curves correspond to the sample exposed to oxygen and the sample blanketed with nitrogen. 


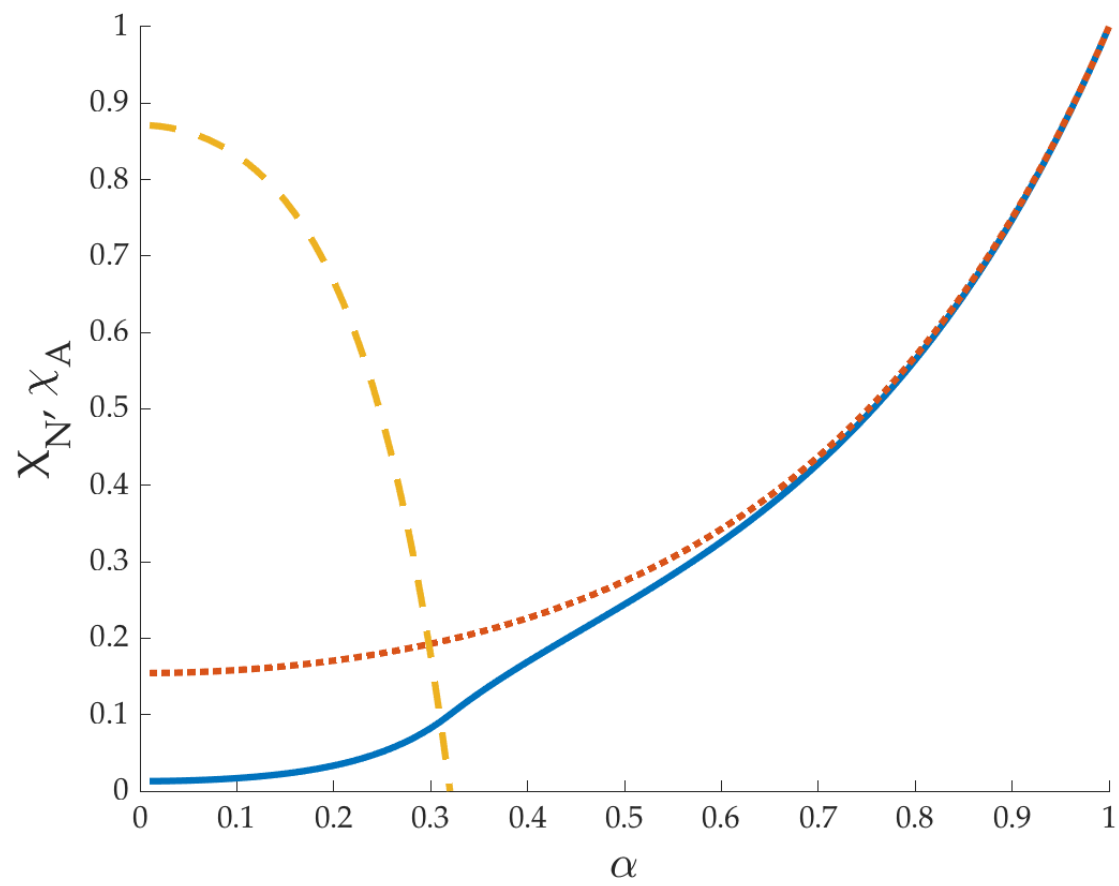

Figure 12: Pseudo-steady state dimensionless antioxidant (dashed) and doublyinflecting oxygen (solid) concentration profiles and the steady state oxygen concentration profile (dotted) versus radius, from Eq. (117)-(120) and Eq. (29), with $\alpha_{I}=0.32$ and dimensionless rate constants of $\kappa=3.93$ and $\kappa_{N A}=13.0$, where $\chi_{N 0}=10$. 


\section{REFERENCES}

${ }^{1}$ Dealy, J.M. and K.F. Wissbrun, “Melt Rheology and Its Role in Plastics Processing: Theory and Applications," Van Nostrand Reinhold, New York (1990).

${ }^{2}$ Giacomin, A.J. and P.H. Gilbert, "Exact-Solution for Cone-Plate Viscometry," Journal of Applied Physics, 122, 175101 (2017), pp. 1-14.

${ }^{3}$ Adams, N., and A. S. Lodge, "Rheological properties of concentrated polymer solutions II. A cone-and-plate and parallel-plate pressure distribution apparatus for determining normal stress differences in steady shear flow," Philosophical Transactions of the Royal Society of London A: Mathematical, Physical and Engineering Sciences, 256 A. 1068, 149-184 (1964).

${ }^{4}$ Schweizer, T., "Comparing cone-partitioned plate and cone-standard plate shear rheometry of a polystyrene melt," Journal of Rheology, 47, 1071 (2003).

${ }^{5}$ Schweizer, T., "Measurement of the first and second normal stress differences in a polystyrene melt with a cone and partitioned plate tool," Rheologica Acta, 41, 337-344 (2002).

${ }^{6}$ Schweizer, T., J. van Meerveld, and H.C. Öttinger, "Nonlinear shear rheology of polystyrene melt with narrow molecular weight distribution-Experiment and theory," Journal of Rheology, 48, 1345-1363 (2004).

${ }^{7}$ Schweizer , T., "A quick guide to better viscosity measurements of highly viscous fluids," Applied Rheology, 14(4), 197-201 (2004).

${ }^{8}$ Snijkers, F. and D. Vlassopoulos, "Cone-partitioned-plate geometry for the ARES rheometer with temperature control," Journal of Rheology, 55 (6), 1167-1186 (2011).

${ }^{9}$ Billingham, N.C., "Degradation," Encyclopedia of Polymer Science and Technology, Concise 3rd Ed., Wiley, 315-321 (2007).

${ }^{10}$ Bird, R.B., W.E. Stewart and E.N. Lightfoot, Transport Phenomena, Revised 2nd ed., Wiley, New York (2007).

${ }^{11}$ Giacomin, A.J., P.H. Gilbert, D. Merger and M. Wilhelm, "Large-Amplitude Oscillatory Shear: Comparing Parallel-Disk with Cone-Plate Flow," Rheologica Acta, 54, 263-285 (2015).

${ }^{12}$ Uhniat, M. and S. Kudła, "Stabilisation of LDPE cross-linked in the presence of peroxides: I. Kinetic study of the oxidation," Polymer Degradation and Stability, 71, 69-74 (2001).

${ }^{13}$ Damköhler, G, "Einflüsse der Strömung, Diffusion und des Wärmeüberganges auf die Leistung von reaktionsöfen.: I. allgemeine Gesichtspunkte für die übertragung eines 
chemischen Prozesses aus dem kleinen ins grosse," Zeitschrift für Elektrochemie und angewandte physikalische Chemie, 42(12), 846-862 (1936). 